\title{
Application of Climate-Driven Model on the Risk of Opisthorchis viverrini Infection in Thailand
}

\author{
Laddawan Jensarikit \\ Department of Mathematics, Faculty of Science \\ King Mongkut's University of Technology Thonburi \\ Bangkok, Thailand
}

Apiporn Suwannatrai

Food-Brone Parasite Research Group, Department of

Parasitology, Faculty of Medicine

Khon Kaen University

Khon Kaen Province 40002, Thailand

\author{
Sanwit Iabchoon \\ KMUTT Geospatial Engineering and Innovation Center \\ (KGEO) \\ King Mongkut's University of Technology Thonburi \\ Bangkok, Thailand
}

\begin{abstract}
Opisthorchis viverrini (O. viverrini), known as a small liver fluke, is a parasite that lives in the bile duct of the mammals including humans. Opisthorchiasis caused by $O$. viverrini infection is a major public health in many countries in Southeast Asia including Thailand and significantly associated with the development of cholangiocarcinoma (bile duct cancer). Environmental factors, particularly climate, significantly influence the life-cycle of $O$. viverrini as well as its intermediate host snails. The climatic parameters such as soil moisture, temperature, and precipitation may alter the geographical distribution of $O$. viverrini by affecting the suitability of freshwater bodies for hosting both parasite and snail populations. Thus, opisthorchiasis is expected to respond to the climate change. However, the influences of climate on the incidence and distribution of opisthorchiasis in Thailand has not yet been addressed. This study aimed to investigate the interaction between climate and opisthorchiasis levels in Thailand using the climate-driven disease model. The climate data and the prevalence of $\boldsymbol{O}$. viverrini infection in 2009 were obtained from the Thai Meteorological Department and Department of Disease Control, Ministry of Public Health, respectively. The risk maps illustrated that the risks of opisthorchiasis in the North and Northeastern regions were found in May, June, July, and September with the highest overall predicted risk being in July. The climate-driven risks of opisthorchiasis in July are consistent with the risks determined from the observed prevalence of $O$. viverrini infection using hot spot analysis. With that, this model can be used to forecast both area and timeframe of opisthorchiasis at the local scale, which can be used to support health planning for implementation of disease prevention and control strategies.
\end{abstract}

Keywords- Opisthorchis viverrini, Climate change, Climatedriven disease model
Pariwate Varnakovida

Department of Mathematics, Faculty of Science, KMUTT Geospatial Engineering and Innovation Center (KGEO)

King Mongkut's University of Technology Thonburi

Bangkok, Thailand

pariwate@gmail.com

\section{INTRODUCTION}

Infection with $O$. viverrini, also known as opisthorchiasis, is a major public health problem in many countries in Southeast Asia including Thailand ${ }^{1,2}$. The liver fluke ( $O$. viverrini) infection in Thailand is variable in each region. Northeastern $(34.6 \%)$ region has the highest prevalence, followed by central $(6.3 \%)$, northern $(5.6 \%)$ and southern $(0.01 \%)$ regions with the nation-wide average prevalence of $14 \%$, or 7 million people were infected across the country ${ }^{11}$. People with an infection have a behavior of eating raw meat of cyprinoid fish, which allows parasites to enter to their bodies. Cyprinoid fish are the second intermediate hosts of $O$. viverrini. Infection of fish occurs by contact with cercariae released from the first intermediate snail host, Bithynia $\mathrm{spp}^{3}$.

Climate plays a significant role in the determination of geographical distribution of $O$. viverrini ${ }^{3,4,5}$. The infection of $O$. viverrini in snail is temperature-dependent in which the increase of $1^{\circ} \mathrm{C}$ resulted in increased odds of infection by $5.4 \%$, whereas the temperature of $34^{\circ} \mathrm{C}$ can be increased the infection rate as $44.14 \%$. Furthermore, there is a report that an annual incidence rate of liver fluke infection in people living in Guangdong City, China corresponded to the increase of temperature and rainfalls ${ }^{6}$. In addition, the distribution of parasites is sensitive to the fluctuations of temperature and precipitation because the water temperature affects sex, size, as well as the survival and reproduction of the snail intermediate hosts of liver flukes ${ }^{6}$.

The prevalence of $O$. viverrini infection in Thailand is changing with close relation to the environmental factors. The 
goal of this study is, thus, to assess the impact of climate changes on $O$. viverrini infection. We first investigated the relationship between climate and the risk of $O$. viverrini infection using the climate-driven disease model. The precision of the model was validated by comparing the model to the risk areas as determined by the hotspot analysis. Taking all the data together, the results from this study will provide better understanding on the possible impact of climate changes on $O$. viverrini infection, which would be beneficial for future policy planning.

\section{METHODOLOGY}

\section{Study location}

Based on the prevalence of $O$. viverrini infection, the Northern and Northeastern regions of Thailand were chosen as the study areas to analyze the risk of $O$. viverrini infection.

\section{Prevalence data of $O$. viverrini infection}

The prevalence data of $O$. viverrini infection in 77 provinces of Thailand in 2009 were obtained from the Department of Disease Control, Ministry of Public Health,Thailand.

\section{Climate data}

Climate data in 2009 were obtained from the Thai Meteorological Department. Monthly data with the following climate variables: minimum, maximum and mean monthly temperature $\left({ }^{\circ} \mathrm{C}\right)$, number of rain days per month $(>1 \mathrm{~mm})$, and monthly rainfall $(\mathrm{mm})$ were used.

\section{The climate-driven disease model analysis}

The climate-driven disease model was used to calculate the risk of opisthorchiasis using the Ollerenshaw index ${ }^{7}$. The monthly $O$. viverrini risk value $(M t)$, which is dependent on the interactions between temperature, precipitation and the frequency of rainy days, was calculated as follows:

$$
M t=n\left(\frac{R}{25.4}-\frac{P}{25.4}+5\right)
$$

(Eq.1)

where $\quad M t=$ Liver fluke risk value (0-100 for a given month), $n=$ Number of rain days per month (defined as day with precipitation more than $1 \mathrm{~mm} /$ day)

$$
\begin{aligned}
& R=\text { Rainfall (mm/month) } \\
& P=\text { Potential evapotranspiration }(\mathrm{mm} / \text { month) }
\end{aligned}
$$

The Hargreaves equation will be used to calculate $(P)^{8}$, where $R \boldsymbol{a}$ is the extra-terrestrial radiation $\left(\mathrm{MJ} \mathrm{m}^{-2}\right.$ day $\left.^{-1}\right)$; with $T_{\max }$ and $T_{\min }$ are the maximum and minimum temperatures in ${ }^{\circ} \mathrm{C}$, respectively.

$$
P=0.0023 \times 0.408 \times R a \times\left(\frac{T_{\max }+T_{\min }}{2}+17.8\right) \times \sqrt{T_{\max }-T_{\min }}
$$

The $M t$ value is defined to zero when the mean monthly temperature is below $10^{\circ} \mathrm{C}$ to reflect the low development thresholds for both the free-living stages of $O$. viverrini and its main snail intermediate host in Thailand ${ }^{9}$. The monthly $M t$ values were applied to forecast for climate-driven risk of opisthorchiasis in 2009. The monthly $M t$ values are classified with four critical thresholds to identify risk as follows: $M t \leq 300$ $=$ no risk; $300<M t \leq 400=$ low risk; $400<M t \leq 474=$ medium risk; $M t>474=$ high risk $^{10}$.

\section{Hot spot analysis}

The location that has statistically significant cluster of opisthorchiasis (risk area) was identified using the hot spot analysis. For hot spot analysis, a Getis-Ord Gi* statistic, which produces a GiZScore and a p-value for each feature, was calculated for each feature according to the properties of its neighbor features using the following formula:

$$
\begin{gathered}
G_{i}^{*}=\frac{\sum_{j=1}^{n} w_{i, j} x_{j}-\bar{X} \sum_{j=1}^{n} w_{i, j}}{S \sqrt{\frac{n \sum_{j=1}^{n} w_{i, j}^{2}-\left(\sum_{j=1}^{n} w_{i, j}\right)^{2}}{n-1}},} \\
\bar{X}=\frac{\sum_{j=1}^{n} x_{j}}{n},
\end{gathered}
$$

(Ep.2)

$$
S=\sqrt{\frac{\sum_{j=1}^{n} x_{j}^{2}}{n}-(\bar{X})^{2}},
$$

(Ep.3)

where

$G_{i}^{*}$ denotes a Getis-Ord Gi* statistic,

$x_{j}$ is the attribute value for feature $j$,

$w_{i j}$ is the spatial weight between feature $i$ and $j$,

and $\quad n$ is equal to the total number of features. 
The results of GiZScore tells features with either high or low values cluster spatially. To be statistically significant hot spot, a feature should have a high value and surrounded by other features with high values. The local sum for a feature and its neighbors is compared proportionally to the sum of all features ${ }^{11}$.

\section{RESULTS AND DISCUSSION}

Predicting the risk of opisthorchiasis using the climate-driven disease model

The predicted risk of opisthorchiasis due to climate in the North and Northeastern regions in 2009 was significantly higher in May, June, July, and September with the highest overall predicted risk in July (Figure 1). From the risk map in July, the provinces with the high risk level (red area) were Bung Kan, Nakhon Phanom, Ubon Ratchathani and Sakon Nakhon, in the Northeastern region. These results indicated that the months when climatic conditions were found to be optimal for the development of the parasite as well as its intermediate host snail in Thailand are May to September, which are the rainy season. This is well in line with several reports demonstrated that rainy season is the most suitable season for the development of the parasite in the environment ${ }^{12}$. In addition, no risk of opisthorchiasis was observed during the dry season (from March to May) and cold season (from November to February). It should be noted that the risk of opisthorchiasis was not found in August and October, which are considered in the rainy season in Thailand. It could possibly be explained by the fact that, in August 2009, there was the low pressure covered the upper part of Thailand resulting in the decrease of precipitation for the
Northern and Northeastern regions. On the contrary, in October 2009 , there was the high pressure covered the upper part of Thailand resulting in the cool weather in the Northern and Northeastern regions.

Predicting the risk of opisthorchiasis using the hot spot analysis

To validate the predicted results obtained from the climatedriven disease model, the hot spot analysis was used to identify clusters of opisthorchiasis based on the prevalence data of $O$. viverrini infection. As shown in Figure 2, the Northeastern region of Thailand has very high clustering (hot spots preseted as red dots) of O.viverrini infection, indicating that this region is at high risk of opisthorchiasis. In contrast, a very high clustering of low O.viverrini infection (cold spots presented as blue spots) was found in the Central, Eastern, and the lower part of Northern regions, indicating that these regions are at low risk of opisthorchiasis. There was no clustering of O.viverrini infection (white spots) in the Western and Southern regions, as there were no observed infection. Furthermore, the hot spots of O.viverrini infection found in Northern region were well in line with the predicted risks of opisthorchiasis based on climatic data. These results indicated that the climate-driven disease model is suitable for forecasting the risk of opisthorchiasis in Thailand.

\section{CONCLUSION}

This study demonstrated that there are strong links between climate and O.viverrini infection, and thus, the climate-driven disease model can be used to assess the impact of recent and potential impact of future climate changes on the risk of opisthorchiasis. 


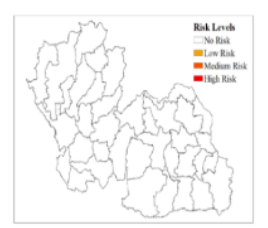

January

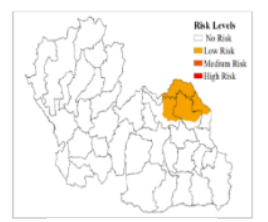

May

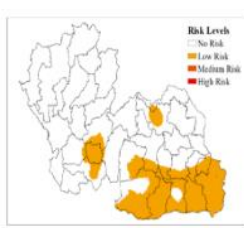

Septembe

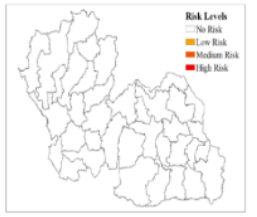

February

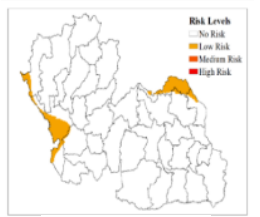

June

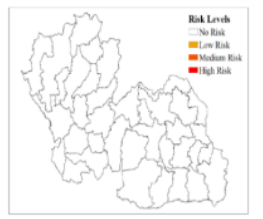

October

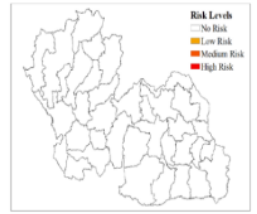

March

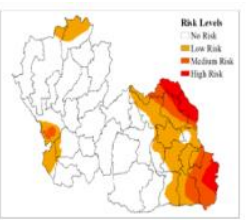

July

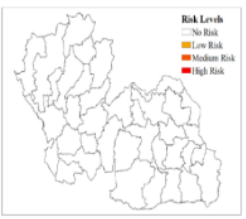

Novembe

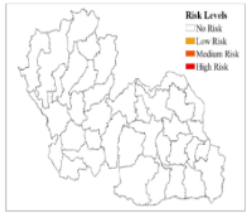

April

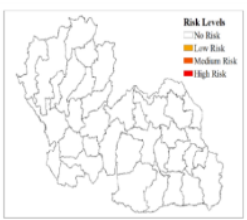

August

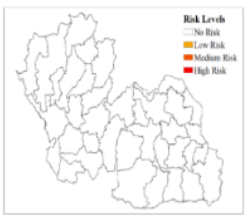

December

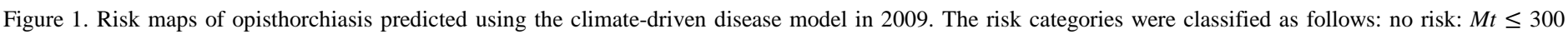
(white), low risk: $300<M t \leq 400$ (brown), medium: $400<M t \leq 474$ (orange), high risk: $M t>474$ (red).

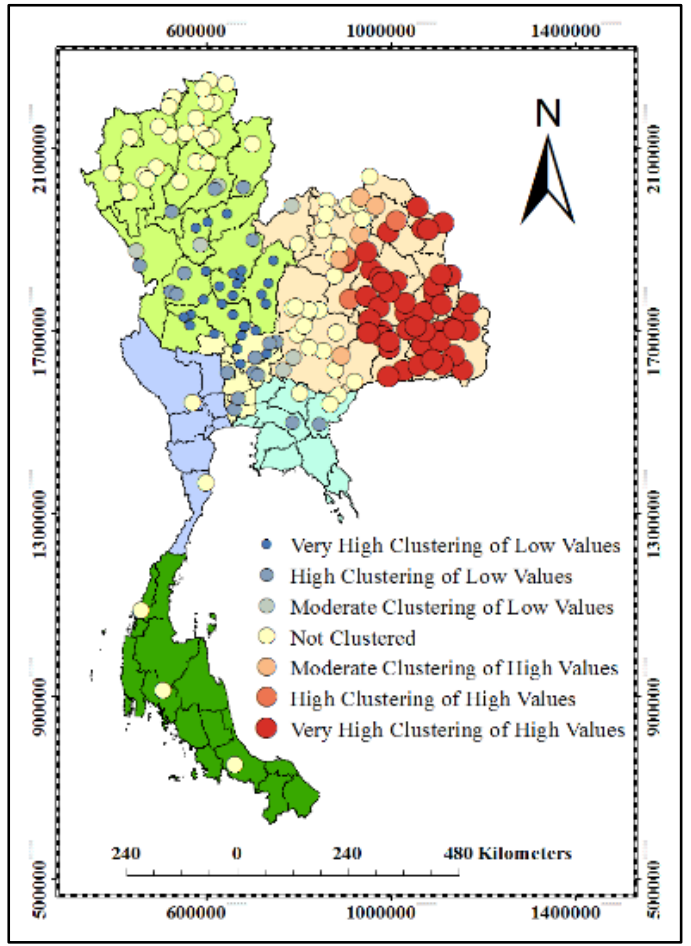

Figure 2. Hot spot analysis using the Getis-Ord Gi* statistics displays the risk of opisthorchiasis in Thailand, 2009.

\section{ACKNOWLEDGEMENTS}

The work was supported by the King Mongkut's of Technology Thonburi. The authors would like to thank Thai Meteorological Department and Department of Disease Control, Prof. Banchob Sripa, and Ministry of Public Health for generously providing the monthly climate data and the prevalence data of O.viverrini infection.

\section{REFERENCES}

[1] Keiser J, Utzinger J. Emerg Infect Dis. 2005; 11(10):1507-1514.

[2] Sithithaworn P, Andrews RH, Nguyen VD, Wongsaroj T, Sinuon M, Odermatt P, Nawa Y, Liang S, Brindley PJ, Sripa B. Parasitol Int. 2012; 61(1): 10-16.

[3] Suwannatrai A, Pratumchart K, Suwannatrai K, Thinkhamrop K, Chaiyos J, Kim CS, Suwanweerakamtorn R, Boonmars T, Wongsaroj T, Sripa B. Parasitol Res. 2017; 116(1):243-250.

[4] Upatham ES, Sukhapanth N. Southeast Asian J Trop Med Public Health. 1980; 11(3):355-358.

[5] Brockelman WY, Upatham ES, Vivanant V, Ardsungnoen S, Chantanawat R. Int J Parasitol. 1986; 16(5): 545-552.

[6] McCreesh N, Nikulin G, Booth M. Parasit Vectors. 2015; 8(4):1-9.

[7] Ollerenshaw CB, Rowlands WT. Veterinary Record. 1959; 71(29):591598.

[8] Hargreaves GH, Samani ZA. Appl Eng Agric. 1985; 1(2):96-99.

[9] Mas-Coma S, Bargues MD, Valero MA. Int J Parasitol. 2005; 35(1112):1255-1278.

[10] Fox NJ, White PCL, McClean CJ, Marion G, Evans A, Hutchings MR. PLoS One. 2011; 6(1):1-9.

[11] Getis A, Ord JK. Geographical Analysis. 1992; 24(3):189-206.

[12] Thai Meteorological Department (TMD). Climate of Thailand (19812010). 1-7. 